\title{
STATE-OF-THE-ART ON MONOLINGUAL LEXICOGRAPHY FOR GREECE (EL)
}

\section{Voula GIOULI, Stella MARKANTONATOU}

Institute for Language and Speech Processing / Athena RIC

Athens, Greece

Giouli, V., Markantonatou, S. (2019). State-of-the-art on monolingual lexicography for Greece (el). Slovenščina 2.o, 7(1): 78-97.

DOI: http://dx.doi.org/10.4312/slo2.0.2019.1.78-97.

The authors report on a recent survey on monolingual dictionaries available on the Greek market. General dictionaries outnumber spelling and educational ones and enjoy a prestigious status. Only one general dictionary is digitally born and only two are available through the web, but several are available as CDs. Most of the prestigious dictionaries have received public funding but not all. Lexicography is well considered in Greece where printed dictionaries seem to still have the lead.

Keywords: monolingual dictionary, market research, lexicography 
This article is a short review of the lexicographic situation in Greece today. We report on an extensive research on the book market and on the bibliographic production of the last decades. Our findings were supplemented with the results of a European survey on dictionary use ${ }^{1}$ (Kosem et al., 2018) that was conducted in the framework of the European Network of e-lexicography (ENeL) COST Action (IS1305) ${ }^{2}$. Being the broadest survey of dictionary use to date, it covers circa 10,000 participants in nearly thirty countries. We aim to offer a detailed picture of the lexicographic landscape in Greece in terms of available resources and their status.

In Section 2 we present our sources of information. Brief information about general, spelling and school monolingual dictionaries is provided in Section 3 and about the format of the dictionaries and the diffusion of internet in Greece in Section 4. In Section 5 we provide some financial information and in Section 6 we discuss our findings concerning the attitude of the public towards Lexicography. The Appendix is an integral part of this document as it contains the three tables with the results of our market search.

\section{SOURCES OF INFORMATION}

We used BIBLIONET as a main source of information. BIBLIONET, a booksin-print database, was developed by the National Book Centre of Greece (EKE$\mathrm{BI}$ ), the main publishers' associations and 45 individual publishers. Currently it is maintained by EKEBI (http://www.gbip.gr). For each book, the database provides updated information about its bibliographic record, market availability and indicative prices. The BIBLIONET listings were checked against information from electronic bookstores and the webpages of major publishing houses.

Additionally, we have drawn information from the national infrastructure DYAS (http://dyas-net.gr/dyas/index.php/en/dyas-project.html). DYAS contributes to the European Infrastructure DARIAH (https://www.dariah.eu/), which aims to connect national infrastructures and incorporate the results of local activities in a common European framework. DYAS lists more than 600 monolingual thesauri.

\footnotetext{
1 http://www.elexicography.eu/events/european-survey-on-dictionary-use/

2 http://www.elexicography.eu/
} 


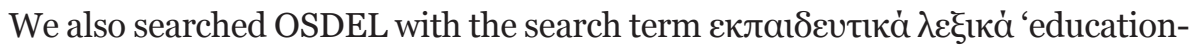
al dictionaries' (http://www.osdelnet.gr). OSDEL is the on-line database of books published in Greece and is maintained by a union of authors, translators and publishers.

Lastly, we searched the proceedings of the International Conference on Greek Linguistics (ICGL), Euralex International Congress (EURALEX), and eLex in the 21st Century in order to find references to the listed dictionaries in scientific publications. We also used a relatively recent study on the state-of-the-art in Greek electronic lexicography (Mantzari and Pantazara, 2014).

\section{MONOLINGUAL LEXICOGRAPHY IN GREECE: MARKET RESEARCH}

The resources showed that 104 general, school, spelling and abbreviation dictionaries of the general Modern Greek language are available on the market right now. Below, we give details about the origins of the general monolingual, spelling, and pedagogical dictionaries, their format and their place in the market.

\subsection{General monolingual dictionaries}

The commercially available general monolingual dictionaries along with year of publication and the name of publisher are listed in Table 1, in the Appendix. 65 . Of them, the dictionaries numbered 3,22,31 are the most highly considered and widely used ones. The ENeL Survey also showed that the dictionary under the number 62 is widely used. The dictionaries under the number 3 and 22 have been produced by the Academy of Athens and the University of Thessaloniki respectively. The dictionary under the number 31 was produced by a private publisher and was supervised by George Babiniotis, Prof. of Linguistics at the University of Athens. Despite their origins, none of the dictionaries numbered 3, 22 and 31 (and no other dictionary) is considered a national symbol according to the ENeL survey results. 62 was produced by a publisher.

\subsection{Spelling dictionaries and their status}

The 14 commercially available spelling dictionaries are listed in table 2 in the Appendix. Almost all major dictionary publishers have released one spelling 
dictionary at least. A tendency for publishing spelling dictionaries just after the release of a general or a school dictionary can be detected in our data suggesting that spelling dictionaries are a by-product of the dictionary development procedure. The ENeL survey shows that spelling dictionaries are in wide use even in the internet era. A possible explanation for this fact might be their size and price. Despite their popularity, however, they do not enjoy the status of the general dictionaries.

\subsection{Special monolingual dictionaries}

The 24 cxommercially available school dictionaries are listed in Table 2 in the Appendix. Since 2002, Greek lexicographic activity has shifted from large-scale, general use dictionaries to specialised pedagogical dictionaries targeted at foreign language learners (Rundell, 1998), (Bullon, 2006) and school students ${ }^{3}$. This is especially true for dictionaries for schools. In 2002, the Ministry of Education and the Pedagogical Institute of Greece initiated a series of dictionaries as part of a national project; they aimed at the reformation of school curricula and the development of teaching material (Gavrilidou et al, 2008). The series consists of two dictionaries for primary school (pupils 6-9 and 10-12 years) and one dictionary for the secondary education students (aged 12-15) compiled on the basis of carefully designed lexicographic and pedagogical specifications. These dictionaries filled the gap in pedagogical lexicography but they have not been updated since then. Their printed versions are distributed free of charge to students in all public schools; they are also available on the market for a fee for students of private schools and all other interested parties. Their electronic versions are freely available as e-books (in pdf format) from the official site of the Greek Ministry of Education.

Dictionaries compiled to suit the needs of foreign learners of Greek are bilingual and fall out of the scope of this enterprise. Only the "Dictionary of Greek

3 We adopt Tarp (2012)'s definition of school dictionary: “a learner's dictionary (or pedagogical dictionary) especially designed to assist school children in learning languages (whether a native or a foreign language) and scientific and practical disciplines”. Consequently, we did not list dictionaries for younger ages, i.e., pre-school children and toddlers, since most of them use a basic vocabulary and pictorial illustrations and short stories instead of definitions. 
as a Foreign Language ${ }^{4}$ " is an available monolingual pedagogical dictionary for school students that learn Greek as a foreign language. It was compiled by the University of Athens in 2007 as part of a national project and it is freely available as an e-book.

\section{DICTIONARY FORMAT}

Nearly all the listed dictionaries are available in paper/book format and only few are offered as a CD-ROM. Sometimes, the CD-ROM edition is available as a stand-alone product at a lower price; others can also be purchased as e-books (in pdf format) but they cannot be adjusted to devices other than personal or portable computers. Only two dictionaries are available on the web: (a) the Dictionary of Standard Modern Greek by Manolis Triantafyllidis ${ }^{5}$ (numbered 22 in Table 1) and (b) the Dictionary of Modern Greek Language ${ }^{6}$ (numbered 10 in Table 1). Dictionary (a) is also available in paper/book format; a digitised version is available via a portal dedicated to the Greek language operated by the Aristotle University of Thessaloniki. The web interface is designed to provide combined access both to other online dictionaries and to textual data. The newly-released dictionary (b) is only browser based, but as yet, it has not been adapted to small screens such as the screen of a tablet or a mobile phone. Only the Small Dictionary of Modern Greek (numbered 33 in Table 1) is currently available as an application for smartphones and tablets.

To the best of our knowledge, only two dictionaries available on the market today are digitally born7: "Synonyms and antonyms Thesaurus of Modern Greek" (Patakis Publications 2005) and the "Dictionary of Modern Greek as a foreign language" (University of Athens, 2007). In both cases, LEXICOGRAPHOS, a language independent software for editing and authoring monolingual and bilingual dictionaries and for publishing dictionaries to hardcopy was employed (Tsalidis et al, 2010). We have not included "Synonyms and Antonyms" in the list with the general dictionaries of Modern Greek.

4 Throughout this document, we use the English title provided by the publisher/developer, where available.

5 http://www.greek-language.gr/greekLang/modern_greek/tools/lexica/triantafyllides/ search.html?lq=

6 http://lexicon-neohel.patakis.gr/el/

7 Neither of them is available on line. 
A note on the internet diffusion in Greece follows with information from two sources. Overall, Greece scores low but not the lowest in the use of internet in Europe.

Internet World Stats (http://www.internetworldstats.com/eu/gr.htm): In Greece, in June 2016 there were 7,072,534 Internet users (65.6\% of the population). Eurostat: In Greece, in 2016, Internet access and use statistics - households and individuals (https://ec.europa.eu/eurostat/statistics-explained/index.php?title=Archive: Internet_access_and_use_statistics_-_households_and_individuals):

1) At EU-Member State level, the lowest proportions of households with access to the internet in Europe were registered in Bulgaria (64\%) and Greece (69\%) as well as the lowest broadband connectivity rates (Bulgaria $63 \%$ and Greece $68 \%$ ).

2) Share of daily users in Greece: $57 \%$.

3) More than one fifth of the population were non-internet users.

\section{FUNDING OF DICTIONARIES AND THE CURRENT BUSINESS MODEL}

Table 1 shows that most of the commercially available dictionaries of Modern Greek have been produced by private enterprises and only two of them by academic units. Nearly all are available as printed books, the exception being dictionary numbered 10 in Table 1 that at the moment is available only as a web service and users have to register before using it.

The average cost of a monolingual Greek dictionary is 24 euros but prices differ significantly. Of the two publicly funded large monolingual dictionaries (numbered 3 and 22 in Table 1), the dictionary under number 3 is available as a printed book at a relatively modest fee given its status and size ${ }^{8}$. The dictionary under number 22, on the other hand, is freely available on the web ${ }^{9}$ but there is no printed version of it. The four pedagogical dictionaries (numbers 13-16 in Table 2) commissioned by the Greek Ministry of Education were publicly funded and are available free of charge for pupils attending public

8 This fact caused the reaction of many columnists, academics and the general public; this reaction was reflected in the press and the digital media.

9 http://www.greeklanguage.gr/greekLang/modern_greek/tools/lexica/triantafyllides/ index.html 
schools. Paying with a subscription is not used as there are few to none dictionaries available on the web or through apps. Dictionary 10 in Table 1 that is the only one available as a web service, can be searched free of charge by registered users but fees for various levels of usage have been announced.

Recently a new business model for the promotion of literary products helped newspapers and publishing houses join forces to increase paper/hard copy press circulation along with advertising and promoting dictionaries: printed versions of dictionaries were offered with the Saturday or the Sunday newspapers at a very low cost. In most cases, special editions were offered such as cheaper editions or series of small volumes with external discriminative features that set them apart from the versions available on the market for a fee.

The Greek audience seems to consider publicly funded dictionaries better than the commercial ones. The commercial dictionaries released by the private-

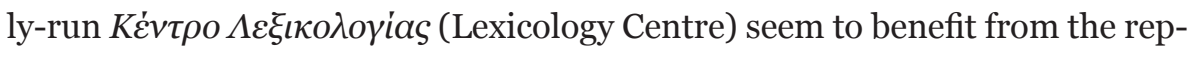
utation owed to Prof. G. Bambiniotis who supervises the lexicographic work.

\section{THE ATTITUDE OF THE GENERAL PUBLIC IN GREECE TO- WARDS DICTIONARIES AND LEXICOGRAPHY}

The ENeL Survey has revealed interesting facts about the attitude of the general public towards dictionaries and lexicography. Despite the fact that Greek is a lesser-spoken language, the number of monolingual dictionaries available on the market is remarkable. However, according to the survey results, dictionaries that are no longer available on the market are still widely used. Dic-

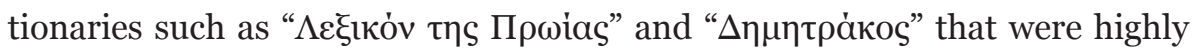
considered during the second half of the 2oth century at least and had a great impact on Greek Lexicography are still in use.

Besides conventional lexicographic resources, survey respondents also opt for Wiktionary and Google Translate. However, this result may be slightly biased because the Wikipedia community of Greece supported the ENeL survey wholeheartedly.

People in Greece do not seem to be really familiar with corpora and corpus use. On the bright side, the portal for the Greek Language at the University of Thessaloniki provides access to textual corpora (journalistic and pedagogical 
texts) and supports combined search in the corpora and the dictionaries hosted. Other corpora ${ }^{10}$ are only known to academics and professionals who work on Language Technologies, Translation and Lexicography and to students. Recently, highly considered private schools have included courses on new technologies but, of course, they are addressed to a minority.

In the ENeL Survey corpora featured in two questions, namely whether users would rank high a dictionary that is corpus-based and/or a dictionary that provides examples extracted from corpora. The criteria were in competition with other desired features of a good dictionary but, despite that, they did not range too low in the responses of the Greek audience.

The general public in Greece is not familiar with electronic lexicography let alone crowdsourcing and its exploitation in lexicography. The younger generation is more familiar with such practices, especially the students - a fact that was also reflected in the European survey on dictionary use.

Lexicography is well-considered in Greece given the following facts:

1) Lexicography has been included as a taught module in most graduate and, especially, post-graduate courses in Greek Universities.

2) The Greek Ministry of Education has financed the compilation of specialized pedagogical dictionaries (discussed in Sections 3-6).

3) Greek universities have been involved in the compilation of dictionaries, for instance the Aristotle University of Thessaloniki and the University of Patras (Kokkinakis et al, 1998).

4) The Academy of Athens has produced and maintains an important dictionary of the general language (numbered 3 in Table 1).

5) There is an important production of (predominantly printed) dictionaries by private enterprises.

6) A private company (Lexicography Center), supervised by Prof. Babiniotis, has produced a series of very popular lexica; of them, the dictionary under number 31 in Table 1 is very prestigious and has become extremely popular.

10 Namely, the Hellenic National Corpus (Hatzigeorgiu et al, 2000) and the Corpus of Greek Texts (Goutsos, 2010). 
The body of research on the use of general dictionaries by the general public in Greece is small. We have consulted the relevant recent publications and have listed them in Section 2. However, these publications only partially cover the field as the first one is about existing electronic dictionaries and the second one only about the use of dictionaries in school. Literature on the use of dic-

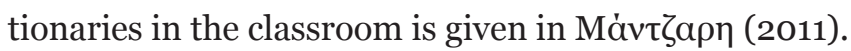

\section{CONCLUSION}

We report on the results of a research focused on monolingual dictionaries in Greece. The main objective of this short review was to present the current situation of Greek lexicography in terms of available resources, format of the dictionaries available on the market and the current business model. The attitude of the general public towards dictionaries and lexicography seems to be rather positive even though alternative reference sources are also employed (ie., Wikipedia). However, the majority of the monolingual dictionaries are still printed rather than digital. In this regard, there is still room for adopting new technologies in lexicography.

\section{ACKNOWLEDGEMENTS}

We acknowledge support of this work by the European Network of e-lexicography (ENeL) COST Action (IS1305).

\section{REFERENCES}

Bullon, S. (2006). The Use of Corpora in Pedagogical Lexicography. In Proceedings of the 13th NIJL International Symposium Language Corpora: Their Compilaltion and Application. Tokyo, Japan. 1-8.

Gavrilidou, M., Giouli, V. and Lambropoulou, P. (2008). The Greek High School Dictionary: Description and issues. In Bernal, E., and DeCesaris, J. (eds.) Proceedings of the XIII Euralex International Congress (EU$R A L E X$ 2008). Barcelona, Spain. Institut Universitari de Linguistica Aplicada, Universitat Pompeu Fabra. 515-524.

Goutsos, D. (2010). The Corpus of Greek Texts: A reference corpus for Modern Greek. Corpora 5(1), 29-44. 
Hatzigeorgiu, N., Gavrilidou, M., Piperidis, S., Carayannis, G., Papakostopoulou, A., Spiliotopoulou, A., Vacalopoulou, A., Labropoulou, P., Mantzari, E., Papageorgiou, H., and Demiros, I. (2000). Design and Implementation of the Online ILSP Greek Corpus. In Gavrilidou et al (eds.) Proceedings of the second international conference of language resources and evaluation (LREC), 1737-1740. European Language Resources Association (ELRA). Athens, Greece.

Kokkinakis, G., Dermatas, E., Koutsogeorgopoulos, E., and Giouli, V.

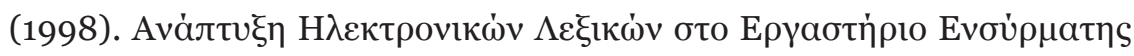

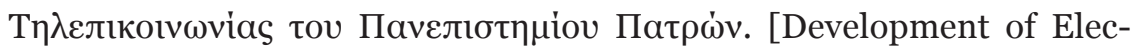
tronic Dictionaries in the Wire Communications Laboratory of the University of Patras]. In Studies for the Greek Language, 19th Annual Meeting of the Linguistics Department of the Aristotle University of Thessaloniki. Institute of Modern Greek Studies. Manolis Triantafyllidis Foundation.

Kosem, I., Lew, R. et al. (2018). The image of the monolingual dictionary across Europe. Results of the European Survey on Dictionary Use and Culture. International Journal of Lexicography 2018 (1), 1-23. doi: 10.1093/ijl/ ecyo22

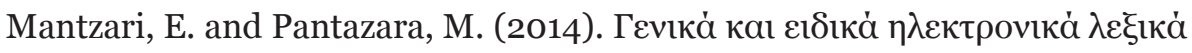

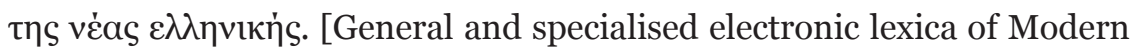
Greek]. In Kotzoglou, K., et al (eds.). Selected Papers of the 11th International Conference on Greek Linguistics, 968-986. Rhodes, University of the Aegean (in Greek).

Rundell, M. (1998). Recent trends in English Pedagogical Lexicography. International Journal of Lexicography 11(4), 315-342.

Tarp, S. (2011). Pedagogical Lexicography: Towards a New and Strict Typology Corresponding to the Present State-of-the-Art. Lexikos. 21(1):217-231. doi: http://dx.doi.org/10.5788/21-1-44

Tsalidis, C., Pantazara, M., Panagiotis, M. and Mantzari, E. (2010). NLP tools for lexicographic applications in Modern Greek. In Granger, S. and Paquot, M. (eds.) Proceedings of the eLex2009: eLexicography in the 21st Century: New Challenges, New Applications. Université Catholique de Louvain. Cahiers du CENTAL No 7, 457-462. 


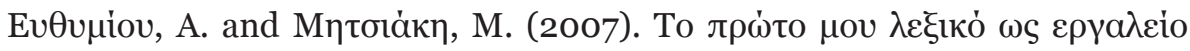

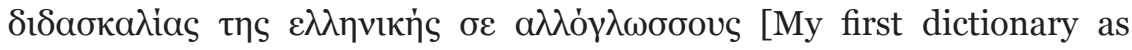
a tool for teaching Greek to non-Greek speakers]. In K. Ntivas and A.

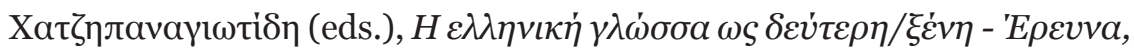

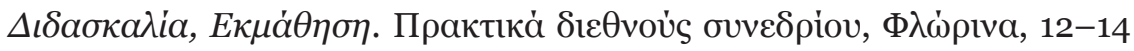
Maïov (2006). University of Western Macedonia. Thessaloniki, University Studio Press.

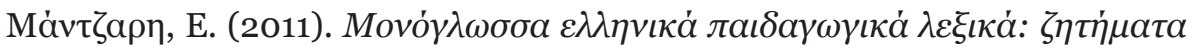

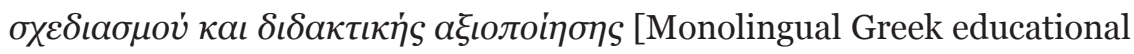
lexica: issues of design and of educational exploitation]. $\mathrm{PhD}$ thesis. University of Patras.

\section{STANJE ENOJEZIČNE LEKSIKOGRAFIJE: GRČIJA}

Prispevek predstavlja nedavno raziskavo o enojezičnih slovarjih, ki so na voljo na grškem trgu. Splošni slovarji so številnejši od pravopisnih in šolskih, hkrati pa imajo prestižnejšo vlogo. Zgolj en splošni slovar je izvorno digitalni, le dva pa sta na voljo na spletu; nekaj jih je na voljo na zgoščenkah. Večina prestižnih slovarjev je bila financirana z javnimi sredstvi in grška leksikografija uživa dober sloves, primat pa imajo tiskani slovarji.

Ključne besede: enojezični slovar, tržna raziskava, leksikogafija

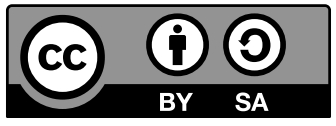

To delo je ponujeno pod licenco Creative Commons: Priznanje avtorstva-Deljenje pod enakimi pogoji 4.o Mednarodna. / This work is licensed under the Creative Commons Attribution-ShareAlike 4.o International.

https://creativecommons.org/licenses/by-sa/4.o/ 


\section{APPENDIX}

Table 1: General dictionaries of Modern Greek.

\begin{tabular}{|c|c|c|c|c|c|c|c|c|c|}
\hline & $\begin{array}{l}\text { Dictionary } \\
\text { title in greek }\end{array}$ & $\begin{array}{l}\text { Dictionary } \\
\text { title translit- } \\
\text { erated }\end{array}$ & Year & $\begin{array}{c}\text { Re- } \\
\text { ferred }\end{array}$ & $\begin{array}{l}\text { Fund- } \\
\text { ed }\end{array}$ & $\begin{array}{l}\text { Print- } \\
\text { ed }\end{array}$ & $\begin{array}{c}\text { Cd } \\
\text { Rom }\end{array}$ & $\begin{array}{c}\text { E- } \\
\text { book }\end{array}$ & $\begin{array}{c}\text { Price } \\
€\end{array}$ \\
\hline 1 & 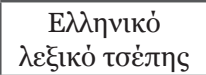 & $\begin{array}{l}\text { Eliniko lexiko } \\
\text { tsepis }\end{array}$ & 2013 & no & $\begin{array}{l}\text { pub- } \\
\text { lisher }\end{array}$ & yes & no & no & 5,6 \\
\hline 2 & 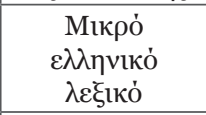 & $\begin{array}{l}\text { Mikro eliniko } \\
\text { lexiko }\end{array}$ & 2007 & no & $\begin{array}{l}\text { pub- } \\
\text { lisher }\end{array}$ & yes & no & no & 8 \\
\hline 3 & 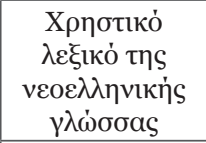 & $\begin{array}{c}\text { Xristiko lexiko } \\
\text { tis neoelinikis } \\
\text { ylosas }\end{array}$ & 2014 & yes & $\begin{array}{l}\text { pub- } \\
\text { licly }\end{array}$ & yes & no & no & 48 \\
\hline 4 & 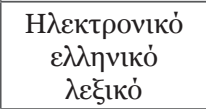 & $\begin{array}{c}\text { Ilektroniko } \\
\text { eliniko lexiko }\end{array}$ & 2009 & yes & $\begin{array}{l}\text { pub- } \\
\text { lisher }\end{array}$ & no & yes & no & 19,9 \\
\hline 5 & 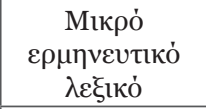 & $\begin{array}{l}\text { Mikro erminef- } \\
\text { tiko lexiko }\end{array}$ & 2009 & no & $\begin{array}{l}\text { pub- } \\
\text { lisher }\end{array}$ & yes & no & no & 10,9 \\
\hline 6 & 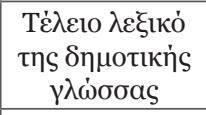 & $\begin{array}{c}\text { Telio lexiko } \\
\text { tis Simotikis } \\
\text { ylosas }\end{array}$ & 1995 & no & $\begin{array}{l}\text { pub- } \\
\text { lisher }\end{array}$ & yes & no & no & 18,66 \\
\hline 7 & 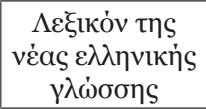 & $\begin{array}{c}\text { Lexikon tis } \\
\text { neas elinikis } \\
\text { ylosis }\end{array}$ & 1971 & no & $\begin{array}{l}\text { pub- } \\
\text { lisher }\end{array}$ & yes & no & no & 145 \\
\hline 8 & 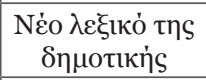 & $\begin{array}{l}\text { Neo lexiko tis } \\
\text { Simotikis }\end{array}$ & 199 & no & $\begin{array}{l}\text { pub- } \\
\text { lisher }\end{array}$ & yes & no & no & 19,7 \\
\hline 9 & 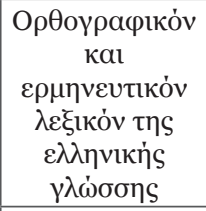 & $\begin{array}{l}\text { Orthografikon } \\
\text { kai erminefti- } \\
\text { kon lexikon tis } \\
\text { elinikis } \gamma \text { losis }\end{array}$ & 1969 & no & $\begin{array}{l}\text { pub- } \\
\text { lisher }\end{array}$ & yes & no & no & 9,59 \\
\hline 10 & 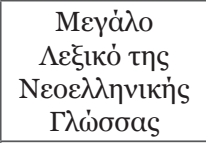 & $\begin{array}{l}\text { Meyalo lexico } \\
\text { tis Neoelinikis } \\
\text { Гlosas }\end{array}$ & 2016 & no & $\begin{array}{l}\text { pub- } \\
\text { lisher }\end{array}$ & no & no & no & \\
\hline 11 & 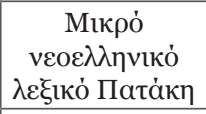 & $\begin{array}{l}\text { Mikro neoe- } \\
\text { liniko lexiko } \\
\text { Pataki }\end{array}$ & 1994 & no & $\begin{array}{l}\text { pub- } \\
\text { lisher }\end{array}$ & yes & no & no & 7,65 \\
\hline 12 & 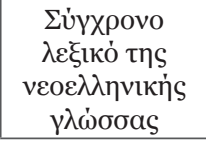 & $\begin{array}{c}\text { Siyxrono lexiko } \\
\text { tis neoelinikis } \\
\text { ylosas }\end{array}$ & 1995 & no & $\begin{array}{l}\text { pub- } \\
\text { lisher }\end{array}$ & yes & no & no & 18,04 \\
\hline
\end{tabular}




\begin{tabular}{|c|c|c|c|c|c|c|c|c|c|}
\hline & $\begin{array}{l}\text { Dictionary } \\
\text { title in greek }\end{array}$ & $\begin{array}{l}\text { Dictionary } \\
\text { title translit- } \\
\text { erated }\end{array}$ & Year & $\begin{array}{c}\text { Re- } \\
\text { ferred }\end{array}$ & $\begin{array}{l}\text { Fund- } \\
\text { ed }\end{array}$ & $\begin{array}{l}\text { Print- } \\
\text { ed }\end{array}$ & $\begin{array}{c}\text { Cd } \\
\text { Rom }\end{array}$ & $\begin{array}{c}\text { E- } \\
\text { book }\end{array}$ & $\begin{array}{l}\text { Price } \\
€\end{array}$ \\
\hline 13 & 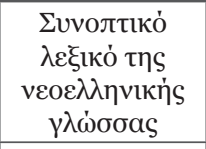 & $\begin{array}{l}\text { Sinoptiko } \\
\text { lexiko tis neoe- } \\
\text { linikis } \gamma \text { losas }\end{array}$ & 1997 & no & $\begin{array}{l}\text { pub- } \\
\text { lisher }\end{array}$ & yes & no & no & 0,5 \\
\hline 14 & 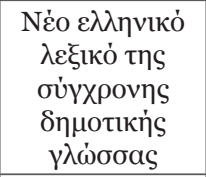 & $\begin{array}{l}\text { Neo lexiko tis } \\
\text { siyxronis Simo- } \\
\text { tikis ylosas }\end{array}$ & 1995 & no & $\begin{array}{l}\text { pub- } \\
\text { lisher }\end{array}$ & yes & no & no & 57,54 \\
\hline 15 & 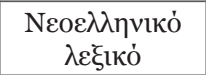 & $\begin{array}{l}\text { Neoeliniko } \\
\text { lexiko }\end{array}$ & 2007 & no & $\begin{array}{l}\text { pub- } \\
\text { lisher }\end{array}$ & yes & no & no & 7,13 \\
\hline 16 & 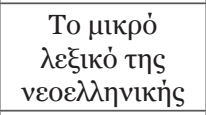 & $\begin{array}{c}\text { To mikro } \\
\text { lexiko tis neoe- } \\
\text { linikis }\end{array}$ & 2001 & no & $\begin{array}{l}\text { pub- } \\
\text { lisher }\end{array}$ & yes & no & no & 4,78 \\
\hline 17 & 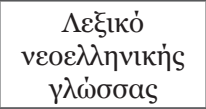 & $\begin{array}{l}\text { Lexiko neoe- } \\
\text { linikis } \gamma \text { losas }\end{array}$ & 2002 & no & $\begin{array}{l}\text { pub- } \\
\text { lisher }\end{array}$ & yes & no & no & 8,3 \\
\hline 18 & 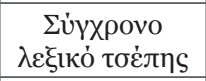 & $\begin{array}{c}\text { Siyxrono lexiko } \\
\text { tsepis }\end{array}$ & 2008 & no & $\begin{array}{l}\text { pub- } \\
\text { lisher }\end{array}$ & yes & no & no & 10,2 \\
\hline 19 & 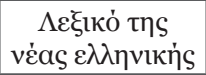 & $\begin{array}{l}\text { Lexiko tis neas } \\
\text { elinikis }\end{array}$ & 2003 & no & $\begin{array}{l}\text { pub- } \\
\text { lisher }\end{array}$ & yes & no & no & 8,59 \\
\hline 20 & 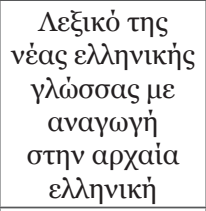 & $\begin{array}{c}\text { Lexiko tis neas } \\
\text { elinikis } \gamma \text { losas } \\
\text { me anayoyi } \\
\text { stin arxea } \\
\text { eliniki }\end{array}$ & 2002 & no & $\begin{array}{l}\text { pub- } \\
\text { lisher }\end{array}$ & yes & no & no & 45,43 \\
\hline 21 & 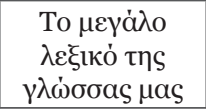 & $\begin{array}{l}\text { To meyalo } \\
\text { lexiko tis ylo- } \\
\text { sas mas }\end{array}$ & 2005 & no & $\begin{array}{l}\text { pub- } \\
\text { lisher }\end{array}$ & yes & no & no & 42,75 \\
\hline 22 & 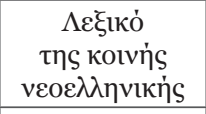 & & 1998 & yes & $\begin{array}{l}\text { pub- } \\
\text { licly }\end{array}$ & yes/no & no & no & 69,24 \\
\hline 23 & 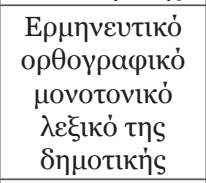 & $\begin{array}{c}\text { Ermineftiko } \\
\text { orӨoyrafiko } \\
\text { monotoniko } \\
\text { lexiko tis Simo- } \\
\text { tikis } \\
\end{array}$ & & no & $\begin{array}{l}\text { pub- } \\
\text { lisher }\end{array}$ & yes & no & no & 7,32 \\
\hline 24 & 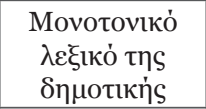 & $\begin{array}{l}\text { Monotoniko } \\
\text { lexiko tis Simo- } \\
\text { tikis }\end{array}$ & 1990 & no & $\begin{array}{l}\text { pub- } \\
\text { lisher }\end{array}$ & yes & no & no & 19,14 \\
\hline 25 & 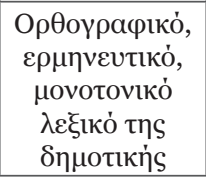 & $\begin{array}{l}\text { OrӨoyrafiko, } \\
\text { monotoniko } \\
\text { lexiko tis Simo- } \\
\text { tikis }\end{array}$ & 1990 & no & $\begin{array}{l}\text { pub- } \\
\text { lisher }\end{array}$ & yes & no & no & 7,17 \\
\hline
\end{tabular}




\begin{tabular}{|c|c|c|c|c|c|c|c|c|c|}
\hline & $\begin{array}{c}\text { Dictionary } \\
\text { title in greek }\end{array}$ & $\begin{array}{l}\text { Dictionary } \\
\text { title translit- } \\
\text { erated }\end{array}$ & Year & $\begin{array}{c}\text { Re- } \\
\text { ferred }\end{array}$ & $\begin{array}{l}\text { Fund- } \\
\text { ed }\end{array}$ & $\begin{array}{l}\text { Print- } \\
\text { ed }\end{array}$ & $\begin{array}{c}\text { Cd } \\
\text { Rom }\end{array}$ & $\begin{array}{c}\text { E- } \\
\text { book }\end{array}$ & $\begin{array}{l}\text { Price } \\
€\end{array}$ \\
\hline 26 & 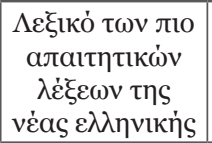 & $\begin{array}{l}\text { Lexiko ton } \\
\text { pio apetitikon } \\
\text { lexeon tis neas } \\
\text { elinikis }\end{array}$ & 2015 & no & $\begin{array}{l}\text { pub- } \\
\text { lisher }\end{array}$ & yes & no & no & 36 \\
\hline 27 & 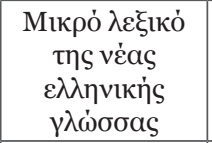 & $\begin{array}{c}\text { Mikro lexiko } \\
\text { tis neas elinikis } \\
\text { ylosas }\end{array}$ & 2006 & yes & $\begin{array}{l}\text { pub- } \\
\text { lisher }\end{array}$ & yes & no & yes & 34 \\
\hline 28 & 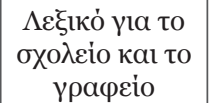 & $\begin{array}{l}\text { Lexiko yia to } \\
\text { sxolio ke to } \\
\text { yrafio }\end{array}$ & 2004 & no & $\begin{array}{l}\text { pub- } \\
\text { lisher }\end{array}$ & yes & no & no & 58 \\
\hline 29 & 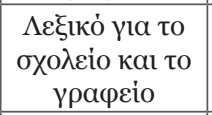 & 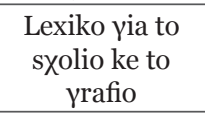 & 2004 & no & $\begin{array}{l}\text { pub- } \\
\text { lisher }\end{array}$ & yes & no & no & o \\
\hline 30 & 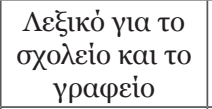 & $\begin{array}{l}\text { Lexiko yia to } \\
\text { sxolio ke to } \\
\text { yrafio }\end{array}$ & 2004 & no & $\begin{array}{l}\text { pub- } \\
\text { lisher }\end{array}$ & yes & no & no & 58 \\
\hline 31 & 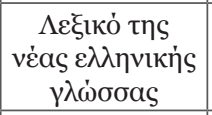 & $\begin{array}{l}\text { Lexiko tis neas } \\
\text { elinikis } \gamma \text { losas }\end{array}$ & 1998 & yes & $\begin{array}{l}\text { pub- } \\
\text { lisher }\end{array}$ & yes & no & no & 96 \\
\hline 32 & 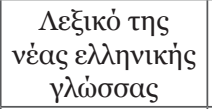 & $\begin{array}{l}\text { Lexiko tis neas } \\
\text { elinikis } \gamma \text { losas }\end{array}$ & 1998 & no & $\begin{array}{l}\text { pub- } \\
\text { lisher }\end{array}$ & yes & no & no & 92 \\
\hline 33 & 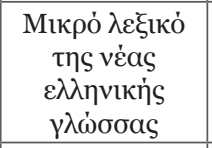 & $\begin{array}{c}\text { Mikro lexiko } \\
\text { tis neas elinikis } \\
\text { ylosas }\end{array}$ & 2013 & no & $\begin{array}{l}\text { pub- } \\
\text { lisher }\end{array}$ & $\begin{array}{c}\text { yes/ } \\
\text { iphone }\end{array}$ & no & no & 34 \\
\hline 34 & 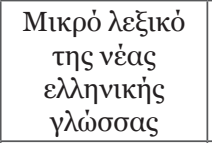 & $\begin{array}{c}\text { Mikro lexiko } \\
\text { tis neas elinikis } \\
\text { ylosas }\end{array}$ & 2006 & no & $\begin{array}{l}\text { pub- } \\
\text { lisher }\end{array}$ & yes & no & no & 28 \\
\hline 35 & 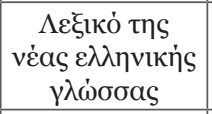 & $\begin{array}{l}\text { Lexiko tis neas } \\
\text { elinikis } \gamma \text { losas }\end{array}$ & 2004 & no & $\begin{array}{l}\text { pub- } \\
\text { lisher }\end{array}$ & yes & no & no & 7,03 \\
\hline 36 & 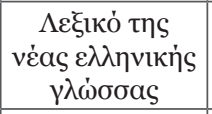 & $\begin{array}{l}\text { Lexiko tis neas } \\
\text { elinikis } \gamma \text { losas }\end{array}$ & 2004 & no & $\begin{array}{l}\text { pub- } \\
\text { lisher }\end{array}$ & yes & no & no & 10,5 \\
\hline 37 & 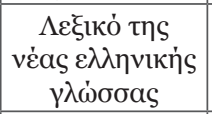 & $\begin{array}{l}\text { Lexiko tis neas } \\
\text { elinikis } \gamma \text { losas }\end{array}$ & 2004 & no & $\begin{array}{l}\text { pub- } \\
\text { lisher }\end{array}$ & yes & no & no & 13,55 \\
\hline 38 & 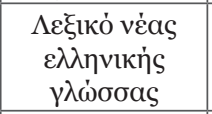 & $\begin{array}{l}\text { Lexiko tis neas } \\
\text { elinikis } \gamma \text { losas }\end{array}$ & 2003 & no & $\begin{array}{l}\text { pub- } \\
\text { lisher }\end{array}$ & yes & no & no & 37,71 \\
\hline 39 & 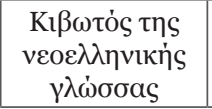 & $\begin{array}{l}\text { Kivotos tis } \\
\text { neoelinikis } \\
\text { ylosas }\end{array}$ & 2009 & no & $\begin{array}{l}\text { pub- } \\
\text { lisher }\end{array}$ & yes & no & no & 47,7 \\
\hline
\end{tabular}




\begin{tabular}{|c|c|c|c|c|c|c|c|c|c|}
\hline & $\begin{array}{l}\text { Dictionary } \\
\text { title in greek }\end{array}$ & $\begin{array}{l}\text { Dictionary } \\
\text { title translit- } \\
\text { erated }\end{array}$ & Year & $\begin{array}{c}\text { Re- } \\
\text { ferred }\end{array}$ & $\begin{array}{l}\text { Fund- } \\
\text { ed }\end{array}$ & $\begin{array}{l}\text { Print- } \\
\text { ed }\end{array}$ & $\begin{array}{c}\text { Cd } \\
\text { Rom }\end{array}$ & $\begin{array}{c}\text { E- } \\
\text { book }\end{array}$ & $\begin{array}{l}\text { Price } \\
€\end{array}$ \\
\hline 40 & 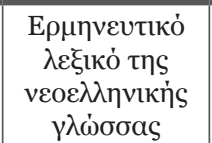 & $\begin{array}{l}\text { Ermineftiko } \\
\text { lexiko tis neoe- } \\
\text { linikis } \gamma \text { losas }\end{array}$ & 2001 & no & $\begin{array}{l}\text { pub- } \\
\text { lisher }\end{array}$ & yes & no & no & 4,64 \\
\hline 41 & 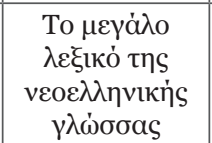 & $\begin{array}{l}\text { To meyalo } \\
\text { lexiko tis neoe- } \\
\text { linikis } \gamma \text { losas }\end{array}$ & 1999 & no & $\begin{array}{l}\text { pub- } \\
\text { lisher }\end{array}$ & yes & no & no & 43,34 \\
\hline 42 & 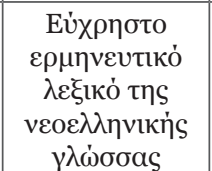 & $\begin{array}{c}\text { Efxristo ermi- } \\
\text { neftiko lexiko } \\
\text { tis neoelinikis } \\
\gamma \text { losas }\end{array}$ & 1998 & no & $\begin{array}{l}\text { pub- } \\
\text { lisher }\end{array}$ & yes & no & no & 18,58 \\
\hline 43 & 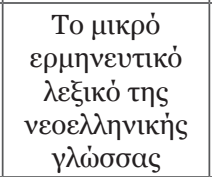 & $\begin{array}{c}\text { To mikro } \\
\text { ermineftiko } \\
\text { lexiko tis neoe- } \\
\text { linikis } \gamma \text { losas }\end{array}$ & 1998 & no & $\begin{array}{l}\text { pub- } \\
\text { lisher }\end{array}$ & yes & no & no & 17,96 \\
\hline 44 & 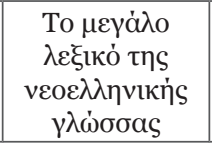 & $\begin{array}{l}\text { To meyalo } \\
\text { lexiko tis neoe- } \\
\text { linikis } \gamma \text { losas }\end{array}$ & 1980 & no & $\begin{array}{l}\text { pub- } \\
\text { lisher }\end{array}$ & yes & no & no & 24,77 \\
\hline 45 & 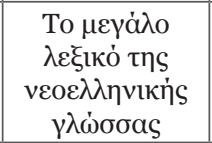 & $\begin{array}{l}\text { To meyalo } \\
\text { lexiko tis neoe- } \\
\text { linikis } \gamma \text { losas }\end{array}$ & 1998 & no & $\begin{array}{l}\text { pub- } \\
\text { lisher }\end{array}$ & yes & no & no & 123,84 \\
\hline 46 & 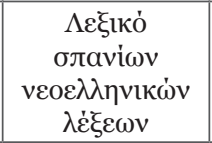 & $\begin{array}{c}\text { Lexiko spanion } \\
\text { neoelinikon } \\
\text { lexeon }\end{array}$ & 2007 & no & $\begin{array}{l}\text { pub- } \\
\text { lisher }\end{array}$ & yes & no & no & 15 \\
\hline 47 & 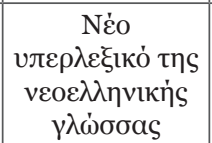 & $\begin{array}{c}\text { Neo iperlexiko } \\
\text { tis neoelinikis } \\
\gamma \text { losas }\end{array}$ & 1999 & yes & $\begin{array}{l}\text { pub- } \\
\text { lisher }\end{array}$ & yes & yes & no & 32,61 \\
\hline 48 & 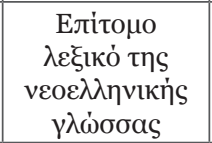 & $\begin{array}{c}\text { Epitomo lexiko } \\
\text { tis neoelinikis } \\
\gamma \text { losas }\end{array}$ & 1992 & no & $\begin{array}{l}\text { pub- } \\
\text { lisher }\end{array}$ & yes & no & no & 29,91 \\
\hline 49 & 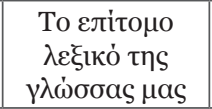 & $\begin{array}{l}\text { To epitomo } \\
\text { lexiko tis } \gamma \text { lo- } \\
\text { sas mas }\end{array}$ & 1991 & no & $\begin{array}{l}\text { pub- } \\
\text { lisher }\end{array}$ & yes & no & no & 10,47 \\
\hline 50 & 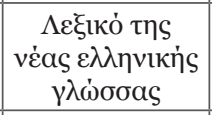 & $\begin{array}{l}\text { Lexiko tis neas } \\
\text { elinikis ylosas }\end{array}$ & 1998 & no & $\begin{array}{l}\text { pub- } \\
\text { lisher }\end{array}$ & yes & yes & no & 71,34 \\
\hline 51 & 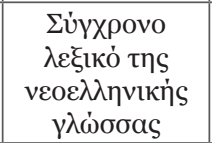 & $\begin{array}{c}\text { Siyxrono lexiko } \\
\text { tis neoelinikis } \\
\text { ylosas }\end{array}$ & 2004 & no & $\begin{array}{l}\text { pub- } \\
\text { lisher }\end{array}$ & yes & no & no & 9,82 \\
\hline
\end{tabular}




\begin{tabular}{|c|c|c|c|c|c|c|c|c|c|}
\hline & $\begin{array}{l}\text { Dictionary } \\
\text { title in greek }\end{array}$ & $\begin{array}{l}\text { Dictionary } \\
\text { title translit- } \\
\text { erated }\end{array}$ & Year & $\begin{array}{c}\text { Re- } \\
\text { ferred }\end{array}$ & $\begin{array}{l}\text { Fund- } \\
\text { ed }\end{array}$ & $\begin{array}{l}\text { Print- } \\
\text { ed }\end{array}$ & $\begin{array}{c}\text { Cd } \\
\text { Rom }\end{array}$ & $\begin{array}{c}\text { E- } \\
\text { book }\end{array}$ & $\begin{array}{l}\text { Price } \\
€\end{array}$ \\
\hline 52 & 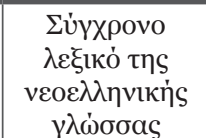 & $\begin{array}{c}\text { Siyxrono lexiko } \\
\text { tis neoelinikis } \\
\text { ylosas }\end{array}$ & 2006 & yes & $\begin{array}{l}\text { pub- } \\
\text { lisher }\end{array}$ & yes & no & no & 24,26 \\
\hline 53 & 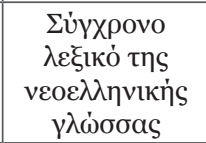 & $\begin{array}{c}\text { Siyxrono lexiko } \\
\text { tis neoelinikis } \\
\text { ylosas }\end{array}$ & 2007 & no & $\begin{array}{l}\text { pub- } \\
\text { lisher }\end{array}$ & yes & no & no & 15,83 \\
\hline 54 & 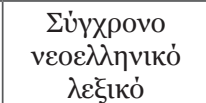 & $\begin{array}{c}\text { Siyxrono neoe- } \\
\text { liniko lexiko }\end{array}$ & 1993 & no & $\begin{array}{l}\text { pub- } \\
\text { lisher }\end{array}$ & yes & no & no & 6,12 \\
\hline 55 & 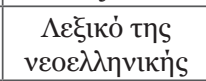 & $\begin{array}{c}\text { Lexiko tis } \\
\text { neoelinikis }\end{array}$ & 1990 & no & $\begin{array}{l}\text { pub- } \\
\text { lisher }\end{array}$ & yes & no & no & 20,19 \\
\hline 56 & 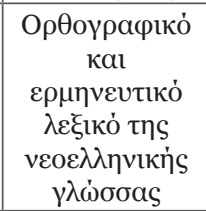 & $\begin{array}{l}\text { OrӨoprafiko ke } \\
\text { ermineftiko } \\
\text { lexiko tis neoe- } \\
\text { linikis } \gamma \text { losas }\end{array}$ & 2004 & no & $\begin{array}{l}\text { pub- } \\
\text { lisher }\end{array}$ & yes & no & no & 20,18 \\
\hline 57 & 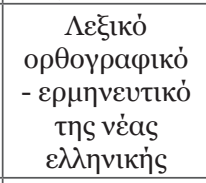 & $\begin{array}{c}\text { Lexico } \\
\text { orӨoyrafiko - } \\
\text { ermineftiko tis } \\
\text { neas elinikis }\end{array}$ & 2011 & no & $\begin{array}{l}\text { pub- } \\
\text { lisher }\end{array}$ & yes & no & no & 9,7 \\
\hline 58 & 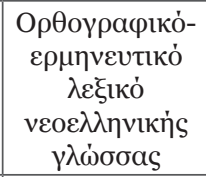 & $\begin{array}{l}\text { OrӨoyrafiko - } \\
\text { ermineftiko } \\
\text { lexiko tis neoe- } \\
\text { linikis } \gamma \text { losas }\end{array}$ & 1997 & no & $\begin{array}{l}\text { pub- } \\
\text { lisher }\end{array}$ & yes & no & no & 8,7 \\
\hline 59 & 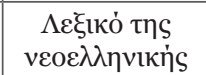 & $\begin{array}{l}\text { Lexiko tis } \\
\text { neoelinikis }\end{array}$ & 1999 & no & $\begin{array}{l}\text { pub- } \\
\text { lisher }\end{array}$ & yes & no & no & 40 \\
\hline 60 & 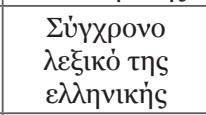 & $\begin{array}{l}\text { Siyxrono lexiko } \\
\text { tis elinikis }\end{array}$ & 1975 & no & $\begin{array}{l}\text { pub- } \\
\text { lisher }\end{array}$ & yes & no & no & 55 \\
\hline 61 & 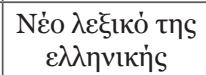 & $\begin{array}{l}\text { Neo lexiko tis } \\
\text { elinikis }\end{array}$ & 1998 & no & $\begin{array}{l}\text { pub- } \\
\text { lisher }\end{array}$ & yes & no & no & 36,68 \\
\hline 62 & 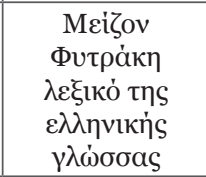 & $\begin{array}{l}\text { Mizon Fitraki } \\
\text { lexiko tis elini- } \\
\text { kis } \gamma \text { losas }\end{array}$ & 2011 & yes & $\begin{array}{l}\text { pub- } \\
\text { lisher }\end{array}$ & yes & no & no & 45 \\
\hline 63 & 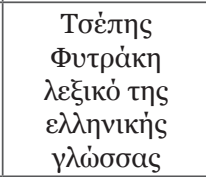 & $\begin{array}{l}\text { Tsepis Fitraki } \\
\text { lexiko tis elini- } \\
\text { kis } \gamma \text { losas }\end{array}$ & 2009 & no & $\begin{array}{l}\text { pub- } \\
\text { lisher }\end{array}$ & yes & no & no & 13 \\
\hline
\end{tabular}




\begin{tabular}{|c|c|c|c|c|c|c|c|c|c|}
\hline & $\begin{array}{l}\text { Dictionary } \\
\text { title in greek }\end{array}$ & $\begin{array}{l}\text { Dictionary } \\
\text { title translit- } \\
\text { erated }\end{array}$ & Year & $\begin{array}{c}\text { Re- } \\
\text { ferred }\end{array}$ & $\begin{array}{l}\text { Fund- } \\
\text { ed }\end{array}$ & $\begin{array}{l}\text { Print- } \\
\text { ed }\end{array}$ & $\begin{array}{c}\text { Cd } \\
\text { Rom }\end{array}$ & $\begin{array}{c}\text { E- } \\
\text { book }\end{array}$ & $\begin{array}{l}\text { Price } \\
€\end{array}$ \\
\hline 64 & 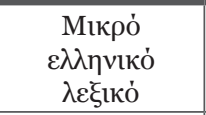 & $\begin{array}{l}\text { Mikro eliniko } \\
\text { lexiko }\end{array}$ & 2009 & no & $\begin{array}{l}\text { pub- } \\
\text { lisher }\end{array}$ & yes & no & no & 28 \\
\hline 65 & 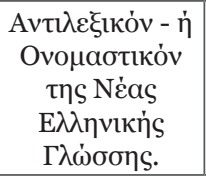 & $\begin{array}{c}\text { Antilexikon i } \\
\text { Onomastikon } \\
\text { tis Neas Elini- } \\
\text { kis Гlosis }\end{array}$ & 2008 & yes & $\begin{array}{l}\text { pub- } \\
\text { lisher }\end{array}$ & yes & no & no & 79,5 \\
\hline
\end{tabular}

Table 2: Spelling dictionaries of Modern Greek.

\begin{tabular}{|c|c|c|c|c|c|c|c|c|c|}
\hline & $\begin{array}{l}\text { Dictionary } \\
\text { title in greek }\end{array}$ & $\begin{array}{l}\text { Dictionary } \\
\text { title translit- } \\
\text { erated }\end{array}$ & Year & $\begin{array}{c}\text { Re- } \\
\text { ferred }\end{array}$ & $\begin{array}{l}\text { Fund- } \\
\text { ed }\end{array}$ & $\begin{array}{l}\text { Print- } \\
\text { ed }\end{array}$ & $\begin{array}{c}\text { Cd } \\
\text { Rom }\end{array}$ & $\begin{array}{c}\text { E- } \\
\text { book }\end{array}$ & $\begin{array}{l}\text { Price } \\
€\end{array}$ \\
\hline 1 & 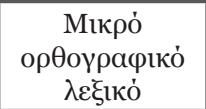 & $\begin{array}{l}\text { Mikro } \\
\text { orӨoyrafiko } \\
\text { lexiko }\end{array}$ & 2008 & & $\begin{array}{l}\text { pub- } \\
\text { lisher }\end{array}$ & yes & no & no & 5,9 \\
\hline 2 & 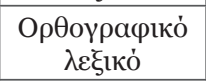 & $\begin{array}{c}\text { OrӨoyrafiko } \\
\text { lexiko }\end{array}$ & 2008 & & $\begin{array}{l}\text { pub- } \\
\text { lisher }\end{array}$ & yes & no & no & 9,9 \\
\hline 3 & 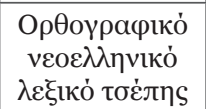 & $\begin{array}{l}\text { OrӨoyrafiko } \\
\text { neoeliniko } \\
\text { lexiko tsepis }\end{array}$ & 1995 & & $\begin{array}{l}\text { pub- } \\
\text { lisher }\end{array}$ & yes & no & no & 6,02 \\
\hline 4 & 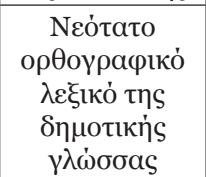 & $\begin{array}{c}\text { Neotato } \\
\text { orӨoyrafiko } \\
\text { lexiko tis Simo- } \\
\text { tikis } \gamma \text { losas }\end{array}$ & 1985 & & $\begin{array}{l}\text { pub- } \\
\text { lisher }\end{array}$ & yes & no & no & 10,65 \\
\hline 5 & 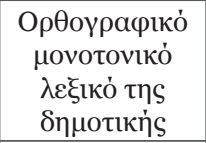 & $\begin{array}{l}\text { OrӨoyrafiko } \\
\text { monotoniko } \\
\text { lexiko tis } \\
\text { Simotikis }\end{array}$ & & & $\begin{array}{l}\text { pub- } \\
\text { lisher }\end{array}$ & yes & no & no & 5,69 \\
\hline 6 & 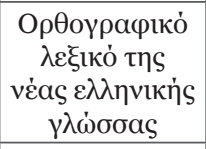 & $\begin{array}{l}\text { OrӨoyrafiko } \\
\text { lexiko tis neas } \\
\text { elinikis ylosas }\end{array}$ & 2008 & & $\begin{array}{l}\text { pub- } \\
\text { lisher }\end{array}$ & yes & no & no & 42 \\
\hline 7 & 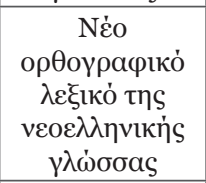 & $\begin{array}{c}\text { Neo } \\
\text { orӨoprafiko } \\
\text { lexiko tis neoe- } \\
\text { linikis } \gamma \text { losas }\end{array}$ & 2005 & & $\begin{array}{l}\text { pub- } \\
\text { lisher }\end{array}$ & yes & no & no & 6,02 \\
\hline 8 & 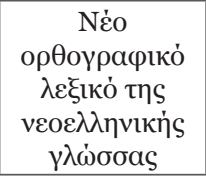 & $\begin{array}{c}\text { Neo } \\
\text { orӨoprafiko } \\
\text { lexiko tis neoe- } \\
\text { linikis } \gamma \text { losas }\end{array}$ & 2002 & & $\begin{array}{l}\text { pub- } \\
\text { lisher }\end{array}$ & yes & no & no & 14,63 \\
\hline
\end{tabular}




\begin{tabular}{|c|c|c|c|c|c|c|c|c|c|}
\hline & $\begin{array}{l}\text { Dictionary } \\
\text { title in greek }\end{array}$ & $\begin{array}{l}\text { Dictionary } \\
\text { title translit- } \\
\text { erated }\end{array}$ & Year & $\begin{array}{c}\text { Re- } \\
\text { ferred }\end{array}$ & $\begin{array}{l}\text { Fund- } \\
\text { ed }\end{array}$ & $\begin{array}{l}\text { Print- } \\
\text { ed }\end{array}$ & $\begin{array}{c}\text { Cd } \\
\text { Rom }\end{array}$ & $\begin{array}{c}\text { E- } \\
\text { book }\end{array}$ & $\begin{array}{l}\text { Price } \\
€\end{array}$ \\
\hline 9 & 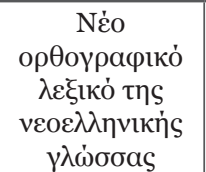 & $\begin{array}{c}\text { Neo } \\
\text { orӨoyrafiko } \\
\text { lexiko tis neoe- } \\
\text { linikis } \gamma \text { losas }\end{array}$ & 1997 & & $\begin{array}{l}\text { pub- } \\
\text { lisher }\end{array}$ & yes & no & no & 6,88 \\
\hline 10 & 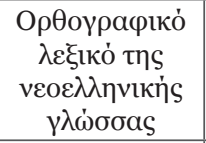 & $\begin{array}{l}\text { OrӨoyrafiko } \\
\text { lexiko tis neoe- } \\
\text { linikis } \gamma \text { losas }\end{array}$ & 1997 & & $\begin{array}{l}\text { pub- } \\
\text { lisher }\end{array}$ & yes & no & no & 6,19 \\
\hline 11 & 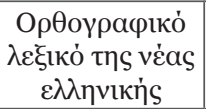 & $\begin{array}{l}\text { OrӨoyrafiko } \\
\text { lexiko tis neas } \\
\text { elinikis }\end{array}$ & 2006 & & $\begin{array}{l}\text { pub- } \\
\text { lisher }\end{array}$ & no & no & yes & 3,9 \\
\hline 12 & 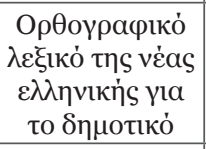 & 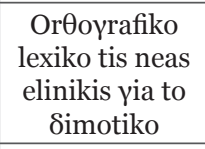 & 2006 & & $\begin{array}{l}\text { pub- } \\
\text { lisher }\end{array}$ & no & no & yes & 3,9 \\
\hline 13 & 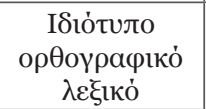 & $\begin{array}{c}\text { ISiotipo } \\
\text { orӨoyrafiko } \\
\text { lexiko }\end{array}$ & 1995 & & $\begin{array}{l}\text { pub- } \\
\text { lisher }\end{array}$ & yes & no & no & 3,05 \\
\hline 14 & 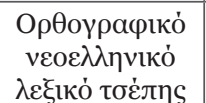 & $\begin{array}{l}\text { OrӨoyrafiko } \\
\text { neoeliniko } \\
\text { lexiko tsepis }\end{array}$ & 1996 & & $\begin{array}{l}\text { pub- } \\
\text { lisher }\end{array}$ & yes & yes & no & 8,44 \\
\hline
\end{tabular}

Table 3: School dictionaries of Modern Greek.

\begin{tabular}{|c|c|c|c|c|c|c|c|c|c|c|}
\hline & $\begin{array}{l}\text { Dictionary title } \\
\text { in greek }\end{array}$ & $\begin{array}{l}\text { Dictionary } \\
\text { title trans- } \\
\text { literated }\end{array}$ & Year & $\begin{array}{c}\text { Ref } \\
\text { Erred }\end{array}$ & $\begin{array}{l}\text { Fund- } \\
\text { ed }\end{array}$ & $\begin{array}{l}\text { Print- } \\
\text { ed }\end{array}$ & \begin{tabular}{|c} 
Cd \\
Rom
\end{tabular} & $\begin{array}{c}\text { E- } \\
\text { Book }\end{array}$ & $\begin{array}{l}\text { Price } \\
\in\end{array}$ & Free \\
\hline 1 & 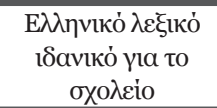 & 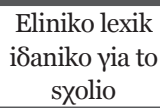 & 2008 & & $\begin{array}{l}\text { pub- } \\
\text { lisher }\end{array}$ & yes & no & no & 14,1 & no \\
\hline 2 & 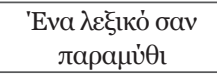 & $\begin{array}{c}\text { Ena lexiko } \\
\text { san parami } \theta \mathrm{i}\end{array}$ & 2009 & & $\begin{array}{l}\text { pub- } \\
\text { lisher }\end{array}$ & yes & no & no & 14,9 & no \\
\hline 3 & 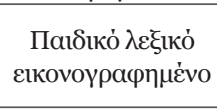 & $\begin{array}{c}\text { Peoiko lexiko } \\
\text { ikonoyrafi- } \\
\text { meno }\end{array}$ & 2009 & & $\begin{array}{l}\text { pub- } \\
\text { lisher }\end{array}$ & yes & no & no & 14,9 & no \\
\hline 4 & 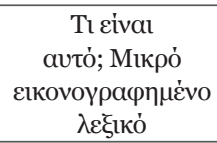 & $\begin{array}{c}\text { Ti ine afto? } \\
\text { Mikro } \\
\text { ikonoyrafi- } \\
\text { meno lexiko }\end{array}$ & 1990 & & $\begin{array}{l}\text { pub- } \\
\text { lisher }\end{array}$ & yes & no & no & 10,2 & no \\
\hline 5 & 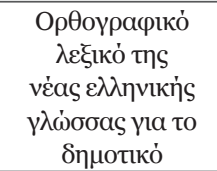 & $\begin{array}{l}\text { OrӨoyrafiko } \\
\text { lexiko tis } \\
\text { neas elinikis } \\
\text { ylosas yia to } \\
\text { Simotiko }\end{array}$ & 2009 & & $\begin{array}{l}\text { pub- } \\
\text { lisher }\end{array}$ & yes & no & no & 5,71 & no \\
\hline
\end{tabular}




\begin{tabular}{|c|c|c|c|c|c|c|c|c|c|c|}
\hline & $\begin{array}{l}\text { Dictionary title } \\
\text { in greek }\end{array}$ & $\begin{array}{l}\text { Dictionary } \\
\text { title trans- } \\
\text { literated }\end{array}$ & Year & $\begin{array}{c}\text { Ref } \\
\text { Erred }\end{array}$ & $\begin{array}{l}\text { Fund- } \\
\text { ed }\end{array}$ & $\begin{array}{l}\text { Print- } \\
\text { ed }\end{array}$ & $\begin{array}{c}\text { Cd } \\
\text { Rom }\end{array}$ & $\begin{array}{c}\text { E- } \\
\text { Book }\end{array}$ & $\begin{array}{l}\text { Price } \\
\boldsymbol{€}\end{array}$ & Free \\
\hline 6 & 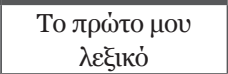 & $\begin{array}{l}\text { To proto mu } \\
\text { lexiko }\end{array}$ & 2001 & & $\begin{array}{l}\text { pub- } \\
\text { lisher }\end{array}$ & yes & no & no & 11,26 & no \\
\hline 7 & 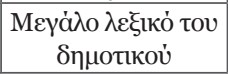 & $\begin{array}{c}\text { Meyalo lexiko } \\
\text { tu Simotiku }\end{array}$ & 2001 & & $\begin{array}{l}\text { pub- } \\
\text { lisher }\end{array}$ & yes & yes & no & $\begin{array}{l}20,00 \\
-6,00\end{array}$ & no \\
\hline 8 & 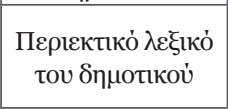 & $\begin{array}{c}\text { Periektiko } \\
\text { lexiko tu } \\
\text { Simotiku }\end{array}$ & 2011 & & $\begin{array}{l}\text { pub- } \\
\text { lisher }\end{array}$ & yes & no & no & 11,45 & no \\
\hline 9 & 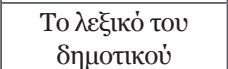 & $\begin{array}{l}\text { To lexiko tu } \\
\text { Simotiku }\end{array}$ & 2001 & yes & $\begin{array}{l}\text { pub- } \\
\text { lisher }\end{array}$ & yes & no & no & 4 & no \\
\hline 10 & 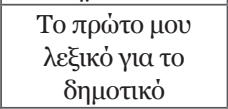 & $\begin{array}{l}\text { To proto mu } \\
\text { lexiko yia to } \\
\text { Simotiko }\end{array}$ & 2000 & & $\begin{array}{l}\text { pub- } \\
\text { lisher }\end{array}$ & yes & no & no & 22,93 & no \\
\hline 11 & 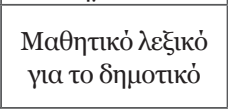 & $\begin{array}{c}\text { MaOitiko } \\
\text { lexiko yia to } \\
\text { Simotiko }\end{array}$ & 2009 & & $\begin{array}{l}\text { pub- } \\
\text { lisher }\end{array}$ & yes & no & no & 17,32 & no \\
\hline 12 & 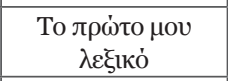 & & 2007 & & $\begin{array}{l}\text { pub- } \\
\text { lisher }\end{array}$ & yes & no & no & 22,82 & no \\
\hline 13 & 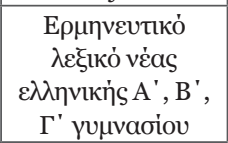 & $\begin{array}{l}\text { Ermineftiko } \\
\text { lexico neas } \\
\text { elinikis A', B;, } \\
\text { Г' Гimnasiu }\end{array}$ & 2008 & yes & publicly & yes & no & no & 8,63 & no \\
\hline 14 & 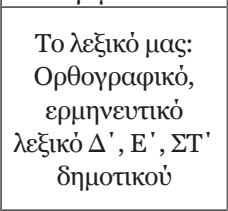 & $\begin{array}{l}\text { To lex- } \\
\text { iko mas: } \\
\text { OrӨoyrafiko, } \\
\text { ermineftiko } \\
\text { lexiko } \Delta^{\prime}, E^{\prime}, \\
\Sigma T^{\prime} \text { Simotiku }\end{array}$ & 2007 & yes & publicly & yes & no & no & 2,88 & no \\
\hline 15 & 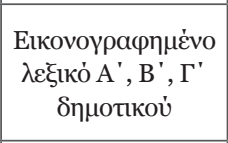 & $\begin{array}{c}\text { Ikonoyrafi- } \\
\text { meno lexiko } \\
\text { A', B', Г' } \\
\text { Simotiku }\end{array}$ & 2007 & yes & publicly & yes & no & no & 5,22 & no \\
\hline 16 & 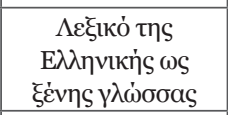 & $\begin{array}{l}\text { Lexiko tis } \\
\text { Elinikis os } \\
\text { xenis } \gamma \text { losas }\end{array}$ & 2007 & yes & publicly & yes & no & yes & o & yes \\
\hline 17 & 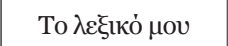 & To lexiko mu & 2005 & & & yes & no & no & 20,29 & no \\
\hline 18 & 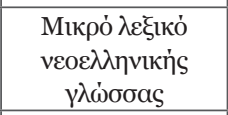 & $\begin{array}{c}\text { Mikro lexiko } \\
\text { neoelinikis } \\
\text { ylosas } \\
\end{array}$ & 1998 & & $\begin{array}{l}\text { pub- } \\
\text { lisher }\end{array}$ & yes & no & no & 11,7 & no \\
\hline 19 & 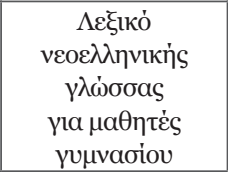 & $\begin{array}{c}\text { Lexiko neoe- } \\
\text { linikis } \gamma \text { losas } \\
\text { yia maӨites } \\
\text { yimnasiu }\end{array}$ & 1997 & & $\begin{array}{l}\text { pub- } \\
\text { lisher }\end{array}$ & & no & no & 10,56 & no \\
\hline
\end{tabular}




\begin{tabular}{|c|c|c|c|c|c|c|c|c|c|c|}
\hline & $\begin{array}{l}\text { Dictionary title } \\
\text { in greek }\end{array}$ & $\begin{array}{l}\text { Dictionary } \\
\text { title trans- } \\
\text { literated }\end{array}$ & Year & $\begin{array}{c}\text { Ref } \\
\text { Erred }\end{array}$ & $\begin{array}{l}\text { Fund- } \\
\text { ed }\end{array}$ & $\begin{array}{l}\text { Print- } \\
\text { ed }\end{array}$ & $\begin{array}{c}\text { Cd } \\
\text { Rom }\end{array}$ & $\begin{array}{c}\text { E- } \\
\text { Book }\end{array}$ & $\begin{array}{c}\text { Price } \\
\boldsymbol{\epsilon}\end{array}$ & Free \\
\hline 20 & 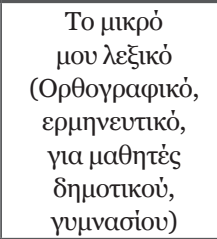 & 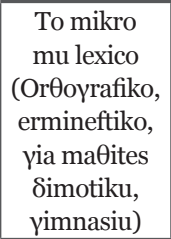 & 2003 & & $\begin{array}{l}\text { pub- } \\
\text { lisher }\end{array}$ & yes & no & no & 8,97 & no \\
\hline 21 & 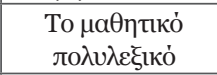 & $\begin{array}{c}\text { To maقitiko } \\
\text { polilexiko }\end{array}$ & 2002 & & $\begin{array}{l}\text { pub- } \\
\text { lisher }\end{array}$ & yes & no & no & 10,1 & no \\
\hline 22 & 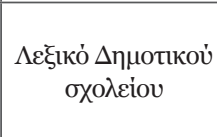 & $\begin{array}{l}\text { Lexiko } \Delta \text { imo- } \\
\text { tiku Sxoliu }\end{array}$ & 2008 & & & yes & yes & no & o & $\begin{array}{c}\text { free ir } \\
\text { news- } \\
\text { pa- } \\
\text { pers }\end{array}$ \\
\hline 23 & 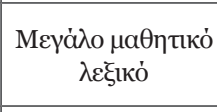 & $\begin{array}{c}\text { Meyalo } \\
\text { maӨitiko } \\
\text { lexiko } \\
\end{array}$ & 2009 & & $\begin{array}{l}\text { pub- } \\
\text { lisher }\end{array}$ & yes & no & no & 9 & no \\
\hline 24 & $\begin{array}{c}\Gamma K I K A ~ \Lambda E \Xi I K O \\
\text { ENNOI } \Omega N \\
\Gamma E N I K H \Sigma \\
\text { ПAI } \Delta \text { EIA } \Sigma\end{array}$ & $\begin{array}{l}\text { GIKA LEX- } \\
\text { IKO ENION } \\
\text { GENIKIS } \\
\text { PE } \triangle \text { IAS }\end{array}$ & 1998 & & $\begin{array}{l}\text { pub- } \\
\text { lisher }\end{array}$ & yes & no & no & 15.85 & no \\
\hline
\end{tabular}

